\title{
An Entanglement Criterion for States in Infinite Dimensional Bipartite Quantum Systems
}

\author{
Yinzhu Wang ${ }^{1,2}$, Danxia Wang ${ }^{2}$ \\ ${ }^{1}$ Department of Mathematics, Taiyuan University of Science and Technology, Taiyuan, China \\ ${ }^{2}$ School of Mathematics, Taiyuan University of Technology, Taiyuan, China \\ Email: 2006wang.yinzhu@163.com
}

Received July 10, 2012; revised August 10, 2012; accepted September 10, 2012

\begin{abstract}
In this paper, an entanglement criterion for states in infinite dimensional bipartite quantum systems is presented. We generalize some of separability criterion that was recently introduced by Wu and Anandan in (Phys. Lett. A, 2002, 297, 4-8) to infinite dimensional bipartite quantum systems. In addition, we give an example aimed to illustrate the application of the theorem.
\end{abstract}

Keywords: Entanglement Criterion; Infinite Dimensional Quantum Systems; Bochner Integral Representation

\section{Introduction}

Quantum entanglement plays a crucial role in the rapidly developing theory of quantum information and quantum computation [1]. In the cases of finite dimensional quantum systems, there are many methods to quantify the entanglement of bipartite and multipartite quantum systems [2-8]. However, most of them have not explicit formula, or it is hard to calculate. For the cases of infinite dimensional systems, the method of entanglement detection is a very difficult problem. But the case of infinite dimensional quantum systems can't be neglected since they do exist in quantum world [9]. Recently, Shengjun $\mathrm{Wu}$ and Jeeva Anandan [10] proposed a necessary criterion based on Pauli matrices re-presentation. Their result is as follows:

Let $H_{A}, H_{B}$ be separable complex Hilbert spaces, $\operatorname{dim} H_{A}=2, \operatorname{dim} H_{B}=n(n<+\infty)$. By $S\left(H_{A} \otimes H_{B}\right)$ we denote the set of all states in $H_{A} \otimes H_{B}$. A given state $\rho$ in $H_{A} \otimes H_{B}$ can be written as:

$$
\rho=\left(\begin{array}{ll}
\rho_{11} & \rho_{12} \\
\rho_{21} & \rho_{22}
\end{array}\right)
$$

where each $\rho_{k l}(k=1,2 ; l=1,2)$ is an $\mathrm{n}$ by $n$ matrix, $\rho$ can also be written as:

$$
\rho=\left(\begin{array}{cc}
M_{0}+M_{z} & M_{x}-i M_{y} \\
M_{x}+i M_{y} & M_{0}-M_{z}
\end{array}\right)
$$

or

$$
\rho=I \otimes M_{0}+\sigma_{x} \otimes M_{x}+\sigma_{y} \otimes M_{y}+\sigma_{z} \otimes M_{z}
$$

where the four matrices

$$
\begin{aligned}
& M_{0}=\frac{1}{2}\left(\rho_{11}+\rho_{22}\right), M_{x}=\frac{1}{2}\left(\rho_{12}+\rho_{21}\right) \\
& M_{y}=\frac{i}{2}\left(\rho_{12}-\rho_{21}\right), M_{z}=\frac{1}{2}\left(\rho_{11}-\rho_{22}\right)
\end{aligned}
$$

are $n$-dimensional Hermitian matrices. Let $R$ be a 3-dimensional real matrix, and $\gamma_{R}$ be a transformation on the density matrix $\rho$ with the following form:

$$
\gamma_{R}(\rho)=\left(\begin{array}{cc}
M_{0}+M_{z}^{R} & M_{x}^{R}-i M_{y}^{R} \\
M_{x}^{R}+i M_{y}^{R} & M_{0}-M_{z}^{R}
\end{array}\right)
$$

where

$$
\left(\begin{array}{c}
M_{x}^{R} \\
M_{y}^{R} \\
M_{z}^{R}
\end{array}\right)=R\left(\begin{array}{l}
M_{x} \\
M_{y} \\
M_{z}
\end{array}\right)
$$

Shengjun $\mathrm{Wu}$ and Jeeva Anandan [10] give the following results:

1) If $\rho$ is separable, then $\gamma_{R}(\rho)$ must be positively defined for any 3-dimensional real matrix $\mathrm{R}$ which satisfies $I-R^{T} R \geq 0$.

2) If $\rho$ is separable, then

a) $M_{0}-\boldsymbol{r} \cdot \boldsymbol{M}$ is positively defined for any vector $\boldsymbol{r}=(x, y, z)$ with $|\boldsymbol{r}| \leq 1, \boldsymbol{M}=\left(M_{x}, M_{y}, M_{z}\right)$;

b) $\operatorname{Tr}\left(M_{0}^{2}-M_{x}^{2}-M_{y}^{2}-M_{z}^{2}\right) \geq 0$; where $M_{x}, M_{y}, M_{z}$ are defined in Equation (4).

Then, a nature problem is arisen: whether or not there is counterpart result for the infinite-dimensional bipartite quantum systems? We find that the answer is "yes". The aim of the present paper is to establish this criterion for the infinite dimensional bipartite quantum systems. 
The paper is organized as follows: In Section 2, we give the main results and the proof of the main results. In Section 3, we give an example to illustrate the application of the theorem.

\section{Some Notations and Main Results}

In this section, we mainly generalize the finite dimensional results, which be proposed by $\mathrm{Wu}$ and Anandan [10], to infinite-dimensional bipartite quantum systems $H_{A} \otimes H_{B}$, where $\operatorname{dim} H_{A}=2, \operatorname{dim} H_{B}=+\infty$.

Let's fix some notations. Let $H_{A}, H_{B}$ be separable complex Hilbert spaces, by $S\left(H_{A} \otimes H_{B}\right)$, we denote the set of all states in $H_{A} \otimes H_{B}$. The set of all separable pure states in $H_{A} \otimes H_{B}$ is denoted by $S_{s-p}\left(H_{A} \otimes H_{B}\right)$. Throughout the paper we use the Dirac's symbols. The bra-ket notation $\langle\cdot \mid \cdot\rangle$ stands for the inner product in the given Hilbert spaces. Recall that a quantum state $\rho \in S\left(H_{A} \otimes H_{B}\right) \quad\left(\operatorname{dim}\left(H_{A} \otimes H_{B}\right)<+\infty\right)$, which is positive and has trace one, is said to be separable if $\rho$ can be written as:

$$
\rho=\sum_{i=1}^{n} p_{i} \rho_{i} \otimes \sigma_{i}
$$

where $\rho_{i}$ and $\sigma_{i}$ are pure states in the subsystems $H_{A}$ and $H_{B}$ respectively, $p_{i} \geq 0, \sum_{i} p_{i}=1$. Otherwise, $\rho_{i}$ is called an entangled state. If $\operatorname{dim}\left(H_{A} \otimes H_{B}\right)=+\infty$, by Werner[8], a state acting on $H_{A} \otimes H_{B}$ is called separable if it can be approximated in the trace norm by the states of the form

$$
\sigma_{n}=\sum_{i=1}^{n} p_{i} \rho_{i} \otimes \sigma_{i}
$$

Furthermore, it is shown in [11] that any separable state $\rho$ admits a representation of the Bochner interal

$$
\rho=\int_{S_{S-p}} \phi\left(\rho^{H_{A}} \otimes \rho^{H_{B}}\right) \mathrm{d} \mu\left(\rho^{H_{A}} \otimes \rho^{H_{B}}\right)
$$

where $\mu$ is a Borel probability measure on

$$
S_{s-p}\left(H_{A} \otimes H_{B}\right) \text {, and } \phi: S_{S-p} \rightarrow S_{S-p}
$$

is a measurable function. It is known that, from the definition of the Bochner integral, there exists a sequences of step function $\left\{\phi_{n}\right\}$, such that

$$
\phi\left(\rho^{H_{A}} \otimes \rho^{H_{B}}\right)=\lim _{n \rightarrow+\infty} \phi_{n}\left(\rho^{H_{A}} \otimes \rho^{H_{B}}\right)
$$

with respect to the trace norm. Where

$$
\phi_{n}\left(\rho^{H_{A}} \otimes \rho^{H_{B}}\right)=\sum_{i=1}^{k_{n}} \chi_{E_{i}}\left(\rho^{H_{A}} \otimes \rho^{H_{B}}\right) \rho_{i}^{H_{A}} \otimes \rho_{i}^{H_{B}}
$$

$\chi_{E_{i}}(\cdot)$ is the characteristic function of $E_{i}$, and $\left\{E_{i}\right\}_{i=1}^{k_{n}}$ is a partition of $S_{s-p}\left(H_{A} \otimes H_{B}\right)$. By $E$ we denote the set of all partitions of $S_{s-p}\left(H_{A} \otimes H_{B}\right)$, Thus we have

$$
\rho=\lim _{E_{i} \in E} \sum_{i} \mu\left(E_{i}\right) \rho_{i}^{H_{A}} \otimes \rho_{i}^{H_{B}}
$$

with respect to the trace norm, as well as with respect to the Hilbert Schmidt norm. Where there exists an ensemble $\left\{p_{i},\left|\psi_{i}^{H_{A}}\right\rangle\right\}$ (or $\left\{p_{j},\left|\varphi_{j}^{H_{B}}\right\rangle\right\}$ ) of $H_{A}$ (or $H_{B}$ ) such that

$$
\rho_{i}^{H_{A}}=\left|\psi_{i}^{H_{A}}\right\rangle\left\langle\psi_{i}^{H_{A}}\right| \quad\left(\text { or } \rho_{i}^{H_{B}}=\left|\phi_{i}^{H_{B}}\right\rangle\left\langle\phi_{i}^{H_{B}}\right|\right)
$$

Next, we give the main results as follows:

Theorem 2.1 Let $H_{A}$ and $H_{B}$ be separable complex Hilbert spaces, $\operatorname{dim} H_{A}=2, \operatorname{dim} H_{B}=+\infty$. If $\rho \in S\left(H_{A} \otimes H_{B}\right)$ is a separable state, then

1) $\gamma_{R}(\rho)$ must be positively defined for any 3 by 3 real matrix $R$ which satisfies $I-R^{T} R \geq 0$, where $\gamma_{R}(\rho)$ is defined in Equations (5) and (6).

2) $M_{0}-\boldsymbol{r} \cdot \boldsymbol{M}$ is positively defined for any vector $\boldsymbol{r}=(x, y, z)$ with $|\boldsymbol{r}| \leq 1, \boldsymbol{M}=\left(M_{x}, M_{y}, M_{z}\right)$ and

$$
\operatorname{Tr}\left(M_{0}^{2}-M_{x}^{2}-M_{y}^{2}-M_{z}^{2}\right) \geq 0, M_{0}, M_{x}, M_{y}, M_{z}
$$

are defined in Equation (4).

Proof. 1) Since $\rho$ is separable, according by Equations (9)-(12), we have

$$
\rho=\lim _{E_{i} \in E} \sum_{i} \mu\left(E_{i}\right)\left|\psi_{i}^{H_{A}}\right\rangle\left\langle\psi_{i}^{H_{A}}|\otimes| \phi_{i}^{H_{B}}\right\rangle\left\langle\phi_{i}^{H_{B}}\right|
$$

where $\left|\psi_{i}^{H_{A}}\right\rangle\left\langle\psi_{i}^{H_{A}}\right|$ are pure states of $H_{A},\left|\varphi_{i}^{H_{B}}\right\rangle\left\langle\varphi_{i}^{H_{B}}\right|$ are pure states of $H_{B}$, respectively. Furthermore, according by Bloch representation [1], we have

$$
\begin{aligned}
& \rho=\lim _{E_{i} \in E} \sum_{i} \mu\left(E_{i}\right) \frac{1}{2}\left(I+\boldsymbol{r}_{i} \cdot \boldsymbol{\sigma}\right) \otimes\left|\phi_{i}^{H_{B}}\right\rangle\left\langle\phi_{i}^{H_{B}}\right| \\
&=\frac{1}{2} \lim _{E_{i} \in E}\left[I \otimes \sum_{i} \mu\left(E_{i}\right)\left|\phi_{i}^{H_{B}}\right\rangle\left\langle\phi_{i}^{H_{B}}\right|\right. \\
&+\sigma_{x} \otimes \sum_{i} \mu\left(E_{i}\right) x_{i}\left|\phi_{i}^{H_{B}}\right\rangle\left\langle\phi_{i}^{H_{B}}\right| \\
&+\sigma_{y} \otimes \sum_{i} \mu\left(E_{i}\right) y_{i}\left|\phi_{i}^{H_{B}}\right\rangle\left\langle\phi_{i}^{H_{B}}\right| \\
&\left.+\sigma_{z} \otimes \sum_{i} \mu\left(E_{i}\right) z_{i}\left|\phi_{i}^{H_{B}}\right\rangle\left\langle\phi_{i}^{H_{B}}\right|\right]
\end{aligned}
$$

with respect to the trace norm, where $\sigma_{x}, \sigma_{y}, \sigma_{z}$ are Pauli matrixes and $\boldsymbol{\sigma}=\left(\sigma_{x}, \sigma_{y}, \sigma_{z}\right), \boldsymbol{r}_{i}=\left(x_{i}, y_{i}, z_{i}\right)$ are real vectors on the Bloch sphere and satisfies $x_{i}^{2}+y_{i}^{2}+z_{i}^{2}=1$. Comparing Equations (3) and (15), we have

$$
\begin{aligned}
& M_{0}=\frac{1}{2} \lim _{E_{i} \in E}\left[\sum_{i=1} \mu\left(E_{i}\right)\left|\phi_{i}^{H_{B}}\right\rangle\left\langle\phi_{i}^{H_{B}}\right|\right] \\
& M_{x}=\frac{1}{2} \lim _{E_{i} \in E}\left[\sum_{i=1} \mu\left(E_{i}\right) x_{i}\left|\phi_{i}^{H_{B}}\right\rangle\left\langle\phi_{i}^{H_{B}}\right|\right]
\end{aligned}
$$




$$
\begin{aligned}
M_{y}= & \frac{1}{2} \lim _{E_{i} \in E}\left[\sum_{i=1} \mu\left(E_{i}\right) y_{i}\left|\phi_{i}^{H_{B}}\right\rangle\left\langle\phi_{i}^{H_{B}}\right|\right] \\
M_{z}= & \frac{1}{2} \lim _{E_{i} \in E}\left[\sum_{i=1} \mu\left(E_{i}\right) z_{i}\left|\phi_{i}^{H_{B}}\right\rangle\left\langle\phi_{i}^{H_{B}}\right|\right] \\
\gamma_{R}(\rho)= & \frac{1}{2} \lim _{E_{i} \in E}\left[I \otimes \sum_{i} \mu\left(E_{i}\right)\left|\phi_{i}^{H_{B}}\right\rangle\left\langle\phi_{i}^{H_{B}}\right|\right. \\
& +\sigma_{x} \otimes \sum_{i} \mu\left(E_{i}\right) x_{i}^{\prime}\left|\phi_{i}^{H_{B}}\right\rangle\left\langle\phi_{i}^{H_{B}}\right| \\
& +\sigma_{y} \otimes \sum_{i} \mu\left(E_{i}\right) y_{i}^{\prime}\left|\phi_{i}^{H_{B}}\right\rangle\left\langle\phi_{i}^{H_{B}}\right| \\
& +\sigma_{z} \otimes \sum_{i} \mu\left(E_{i}\right) z_{i}^{\prime}\left|\phi_{i}^{H_{B}}\right\rangle\left\langle\phi_{i}^{H_{B}}\right|
\end{aligned}
$$

where

$$
\left(\begin{array}{c}
x_{i}^{\prime} \\
y_{i}^{\prime} \\
z_{i}^{\prime}
\end{array}\right)=R\left(\begin{array}{c}
x_{i} \\
y_{i} \\
z_{i}
\end{array}\right)
$$

Since for any 3-dimensional real matrix $R$ which satisfies $I-R^{T} R \geq 0$, means that $\left(x_{i}^{\prime}\right)^{2}+\left(y_{i}^{\prime}\right)^{2}+\left(z_{i}^{\prime}\right)^{2} \leq 1$, on the other hand, according by Equation (16), we have

$$
\gamma_{R}(\rho)=\frac{1}{2} \lim _{E_{i} \in E}\left[A \otimes \sum_{i} \mu\left(E_{i}\right)\left|\phi_{i}^{H_{B}}\right\rangle\left\langle\phi_{i}^{H_{B}}\right|\right],
$$

where

$$
A=\left(\begin{array}{cc}
1+z_{i}{ }^{\prime} & x_{i}{ }^{\prime}-i y_{i}{ }^{\prime} \\
x_{i}{ }^{\prime}+i y_{i}{ }^{\prime} & 1-z_{i}{ }^{\prime}
\end{array}\right)
$$

It is obvious that we have $A \geq 0$, in fact since $\left(x_{i}^{\prime}\right)^{2}+\left(y_{i}^{\prime}\right)^{2}+\left(z_{i}^{\prime}\right)^{2} \leq 1$, so $\gamma_{R}(\rho)$ is still a density matrix, i.e., $\gamma_{R}(\rho) \geq 0$.

$$
\text { 2) } \begin{aligned}
& M_{0}-\boldsymbol{r} \cdot \boldsymbol{M} \\
= & M_{0}-x M_{x}-y M_{y}-z M_{z} \\
= & \frac{1}{2} \lim _{E_{i} \in E}\left[\sum_{i} \mu\left(E_{i}\right)\left|\phi_{i}^{H_{B}}\right\rangle\left\langle\phi_{i}^{H_{B}}\right|\right] \\
& -x \frac{1}{2} \lim _{E_{i} \in E}\left[\sum_{i=1} \mu\left(E_{i}\right) x_{i}\left|\phi_{i}^{H_{B}}\right\rangle\left\langle\phi_{i}^{H_{B}}\right|\right] \\
& -y \frac{1}{2} \lim _{E_{i} \in E}\left[\sum_{i=1} \mu\left(E_{i}\right) y_{i}\left|\phi_{i}^{H_{B}}\right\rangle\left\langle\phi_{i}^{H_{B}}\right|\right] \\
& -z \frac{1}{2} \lim _{E_{i} \in E}\left[\sum_{i} \mu\left(E_{i}\right) z_{i}\left|\phi_{i}^{H_{B}}\right\rangle\left\langle\phi_{i}^{H_{B}}\right|\right] \\
= & \frac{1}{2} \lim _{E_{i} \in E}\left[\sum_{i} \mu\left(E_{i}\right) z_{i}\left|\phi_{i}^{H_{B}}\right\rangle\left\langle\phi_{i}^{H_{B}}\right|\right]\left[1-x x_{i}-y y_{i}-z z_{i}\right]
\end{aligned}
$$

Since $|\boldsymbol{a} \cdot \boldsymbol{b}| \leq|\boldsymbol{a}| \cdot|\boldsymbol{b}|$, so we have $1-x x_{i}-y y_{i}-z z_{i} \geq 0$ and $M_{0}-\boldsymbol{r} \cdot \boldsymbol{M} \geq 0$.

On the other hand, we have

$$
\begin{aligned}
\operatorname{Tr}\left(M_{0}^{2}-M_{x}^{2}-M_{y}^{2}-M_{z}^{2}\right) \\
=\frac{1}{4} \lim _{E_{i} \in E}\left[\sum_{i} \sum_{j} \mu\left(E_{i}\right) \mu\left(E_{j}\right)\right. \\
\left.\times\left(1-x_{i} x_{j}-y_{i} y_{j}-z_{i} z_{j}\right)\left|\left\langle\phi_{i}^{H_{B}} \mid \phi_{i}^{H_{B}}\right\rangle\right|^{2}\right] \geq 0
\end{aligned}
$$

This completes the proof.

\section{Example}

Next, we give an example to illustrate the application of the Theorem 2.1. We consider a bipartite infinite dimensional state with the following forms:

$$
\rho=x\left|00^{\prime}\right\rangle\left\langle 00^{\prime}\right|+\frac{1-x}{2}\left(\left|01^{\prime}\right\rangle+\left\langle 10^{\prime}\right|\right)\left(\left\langle 01^{\prime}\right|+\left\langle 10^{\prime}\right|\right)
$$

with $\rho \in S\left(H_{A} \otimes H_{B}\right)$

$$
\operatorname{dim} H_{A}=2, \operatorname{dim} H_{B}=+\infty, \quad 0 \leq x \leq 1,
$$

where $\{|0\rangle,|1\rangle\}$ is the orthogonal real basis of $H_{A}$,

$$
\left\{\left|0^{\prime}\right\rangle,\left|1^{\prime}\right\rangle, \cdots\right\} \text { is the orthogonal real basis of } H_{B} .
$$

For simplicity, we assume $\operatorname{dim} H_{B}=n(2 \leq n \leq+\infty)$. In this case, we can obtain that

$$
\begin{aligned}
& M_{0}=\left(\begin{array}{cccc}
\frac{1+x}{4} & 0 & \cdots & 0 \\
0 & \frac{1-x}{4} & \cdots & 0 \\
0 & 0 & &
\end{array}\right) \\
& M_{x}=\left(\begin{array}{cccc}
0 & \frac{1-x}{4} & \cdots & 0 \\
\frac{1-x}{4} & 0 & \cdots & 0 \\
0 & 0 & &
\end{array}\right) \\
& M_{y}=\frac{i}{2}\left(\begin{array}{cccc}
0 & \frac{x-1}{2} & \cdots & 0 \\
\frac{1-x}{2} & 0 & \cdots & 0 \\
0 & 0 & &
\end{array}\right) \\
& M_{z}=\left(\begin{array}{cccc}
\frac{3 x-1}{4} & 0 & \cdots & 0 \\
0 & \frac{1-x}{4} & \cdots & 0 \\
0 & 0 & &
\end{array}\right)
\end{aligned}
$$


According by Theorem 2.1, by a straightforward calculation, we obtain the following result, if

$$
0<x<\frac{1}{5}
$$

then

$$
\operatorname{Tr}\left(M_{0}^{2}-M_{x}^{2}-M_{y}^{2}-M_{z}^{2}\right)<0
$$

so $\rho$ is entangled.

Remark: It is obvious that this criterion of Theorem 2.1 is weaker than PPT criterion [4], in fact if $x \neq 1, \rho$ is also entangled, but this criterion give us a method to detect the entanglement for states in infinite bipartite quantum systems.

\section{Acknowledgements}

This work was partially supported by the National Natural Science Foundation of China (11171249) and the Natural Science Foundation of Shanxi Province (2011021002-2). The authors also wish to give their thanks to the referees for their comments to improve the presentation of this paper.

\section{REFERENCES}

[1] M. A. Nielsen and I. L. Chang, "Quantum Computation and Quantum Information," Cambridge University press, Cambridge, 2000.

[2] A. Peres, "Separability Criterion for Density Matrices," Physical Review Letters, Vol. 77, No. 8, 1996, pp. 14131418. doi:10.1103/PhysRevLett.77.1413

[3] K. Zyczkowski, P. Horodecki, A. Sanpera, et al., "Volume of the Set of Separable States," Physical Review A,
Vol. 58, No. 2, 1998, pp. 883-896. doi:10.1103/PhysRevA.58.883

[4] M. Horodecki, P. Horodecki and R. Horodecki, "Separability of Mixed States: Necessary and Sufficient Conditions," Physical Review A, Vol. 223, No. 1-2, 1996, pp. 1-14. doi:10.1016/S0375-9601(96)00706-2

[5] M. Horodecki and P. Horodecki, "Reduction Criterion of Separability and Limits for a Class of Distillation Protocols," Physical Review A, Vol. 59, No. 6, 1999, pp. 42064216. doi:10.1103/PhysRevA.59.4206

[6] M. A. Nielsen and J. Kempe, "Separable States Are More Disordered Globally than Locally," Physical Review Letters, Vol. 86, No. 22, 2001, pp. 5184-5187. doi:10.1103/PhysRevLett.86.5184

[7] O. Rudolph, "Further Results on the Cross Norm Criterion for Separability," Quantum Information Processing, Vol. 4, No. 3, 2005, pp. 219-239. doi:10.1007/s11128-005-5664-1

[8] R. F. Werner, "Quantum States with Einstein-PodolskyRosen Correlations Admitting a Hidden-Variable Model," Physical Review A, Vol. 40, No. 8, 1989, pp. 4277-4281. doi:10.1103/PhysRevA.40.4277

[9] X. L. Su, X. J. Jia, C. D. Xie, et al., "Generation of GHZ-Like and Duster-Like Quadripripartite Entangled States for Continuous Variable Using a Set of Quadrature Squeezed States," Science in China Series G: Physics Mechanics and Astronomy, Vol. 51, No. 1, 2008, pp. 1-13. doi:10.1007/s11433-008-0004-y

[10] S. J. Wu and J. Anandan, "Some Aspects of Separability," Physical Review A, Vol. 297, No. 1-2, 2002, pp. 4-8. doi:10.1016/S0375-9601(02)00279-7

[11] A. S. Holevo, M. E. Shirokov and R. F. Werner, "Separability and Entanglement-Breaking in Infinite Dimensionals," Russian Math Surveys, Vol. 60, No. 2, 2005, pp. $1-12$. 